\title{
Síndrome cardio-renal anémico
}

\section{Cardio-renal anemia syndrome}

\author{
Ana Cristina Montenegro, luis Eduardo Silva, María Angélica Muñoz \\ - Bogotá, D.C. (Colombia)
}

\section{Resumen}

Las interacciones entre el corazón y el riñón se han convertido en un área de considerable interés, dada la interdependencia de los mismos. Esto motivó la definición y conceptualización del síndrome cardio-renal anémico, que incluye interacciones bidireccionales, donde alteraciones, tanto agudas como crónicas de cualquier órgano, pueden afectar indistintamente la función renal o la ventricular. El tratamiento, involucra el bloqueo del eje renina angiotensina aldosterona y durante una descompensación aguda es válido el soporte dialítico para control de la volemia. La anemia, es multifactorial, se debe tratar de manera oportuna con hierro endovenoso y eritropoyetina recombinante, reduciendo al mínimo el soporte transfusional. El manejo, la definición y el pronóstico del síndrome cardio-renal anémico aún sigue siendo controversial y es un reto para el internista moderno (Acta Med Colomb 2011; 36: 141-144).

Palabras clave: insuficiencia cardiaca, insuficiencia renal, síndrome cardio-renal anémico, eritropoyetina recombinante, hierro endovenoso, soporte dialítico.

\section{Abstract}

The interactions between the heart and the kidney have become an area of considerable interest, given their interdependence. This led to the definition and conceptualization of cardio-renal anemic syndrome, which includes a bidirectional interaction: acute or chronic injuries of any organ indiscriminately affect renal or ventricular function. Treatment involves blocking the rennin-angiotensinaldosterone axis and, during acute decompensation, dialytic support for volemic control. Anemia is due to multiple causesand must be treated promptly with intravenous iron and recombinant erythropoietin, to minimize the need for transfusions. Management, definition and prognosis of cardio-renal anemic syndrome are still controversial and represent a challenge for the modern internist (Acta Med Colomb 2011; 36: 141-144).

Key words: cardiac failure, renal failure, cardio-renal anemic syndrome, recombinant erythropoietin, intravenous iron, dialysis support.

Dra. Ana Cristina Montenegro: Internista Institucional, Hospital Universitario Fundación Santa Fe de Bogotá, (FSFB); Dr. Luis Eduardo Silva: Interno Universidad de Los Andes-FSFB; Dra. María Angélica Muñoz: Interno Universidad de los Andes-FSFB. Departamento de Medicina Interna FSFB Bogotá, D.C. (Colombia).

Correspondencia. Dra. Ana Cristina Montenegro. Bogotá, D.C. (Colombia).

E-mail: montenegroarenas@gmail.com Recibido: 27/XII/2010 Aceptado: 14/VII/2011

\section{Reporte de caso}

Paciente de 67 años, con antecedentes de insuficiencia cardiaca (IC) estadio C de la clasificación del Colegio Americano de Cardiología (ACC), diabetes mellitus tipo 2 (DM2), enfermedad pulmonar obstructiva crónica (EPOC), hipertensión arterial (HTA), anemia microcítica hipocrómica con ferropenia severa, insuficiencia renal crónica (IRC) estadio III, insuficiencia y estenosis leve de válvula mitral, insuficiencia tricuspídea severa, quien consultó por cuadro clínico de cuatro meses de evolución consistente en edema de miembros inferiores, distensión abdominal marcada secundaria a ascitis, disnea clase funcional III/IV de la NYHA (New York Heart Association), cuadro por el que había requerido múltiples hospitalizaciones. Al examen físico se encuentra ingurgitación yugular grado 3 evaluada a $45^{\circ}$, tensión arterial de $160 / 80 \mathrm{mmHg}$, a la auscultación cardiaca se documentan ruidos cardiacos rítmicos con soplo holosistólico grado IV/VI en foco tricuspídeo, que aumenta con la inspiración, con crépitos finos bibasales documentados como único hallazgo en la auscultación pulmonar, abdomen distendido, con onda ascítica presente, doloroso a la palpación generalizada, edema grado 3 de miembros inferiores, saturando $96 \%$ con oxígeno al aire ambiente. Dentro de los paraclínicos solicitados, los de mayor relevancia se encuentran en las Tablas 1 y 2 .

La paciente es transfundida en varias ocasiones. Presenta retención progresiva de líquidos con disminución de función renal que pasó de tasa de filtración glomeru- 
$\operatorname{lar}$ (TFG) de $43 \mathrm{~mL} / \mathrm{min} / 1.73 \mathrm{~m}^{2}$ a $28 \mathrm{~mL} / \mathrm{min} / 1.73 \mathrm{~m}^{2}$ calculadas por fórmula MDRD (Modification of Diet in Renal Disease formula, obtenida de American Journal of Kidney Disease 2002;39 (Suppl1):S1) con resistencia a manejo con diuréticos. El encontrar falla cardiaca, junto a insuficiencia renal crónica de origen hipertensiva, anemia y resistencia a diuréticos se considera como primera opción síndrome cardio-renal anémico (SCRA) tipo 2. Se traslada a la unidad de cuidado intensivo para manejo integral, con énfasis en la hipervolemia con requerimiento de inotropía, monitoreo de presión venosa central, furosemida intravenosa (IV) e hidroclorotiazida oral, albumina y hierro IV, sin requerimiento de terapia dialítica gracias al manejo integral. Tras una adecuada evolución, se da de alta con omeprazol, eritropoyetina recombinante, hidroclorotiazida, espironolactona, losartan, furosemida, hierro, insulina NPH y metoprolol.

\section{Discusión}

Un alto porcentaje de pacientes hospitalizados presentan disfunción cardiaca y renal concomitantemente: los novedosos tratamientos para las enfermedades crónicas y el envejecimiento de la población con un deficiente control de los factores de riesgo tradicionales, son las principales explicaciones para su creciente incidencia. El vínculo existente entre el riñón y el corazón ha sido estudiado ampliamente: la presencia de disfunción renal en pacientes con cardiopatía sintomática o asintomática, la concepción de la nefropatía como factor de riesgo independiente para enfermedad cardiovascular y su interdependencia tanto fisiológica como patológicamente, constituyen los factores de convergencia de este nuevo síndrome (1-5). El síndrome cardio-renal anémico (SCRA) es una condición que involucra la compleja red de interacciones bidireccionales de la función cardiaca y renal: en la noxa inicial, una alteración tanto aguda como crónica cardiaca (SCRA tipo 1, 2) genera un impacto negativo a largo o corto plazo en el funcionamiento del segundo órgano. Así mismo, se puede concebir el síndrome renocardiaco (SRCA tipo 3 y 4) cuando la noxa inicial se documenta en el funcionamiento renal, tanto agudo como crónico, conllevando una alteración
Tabla 1. Analítica sanguínea.

\begin{tabular}{|ll|}
\hline Cuadro hemático & $\begin{array}{l}\text { Leucocitos: } 8070 \text {, neutrófilos: } 70 \% \text {, linfocitos: } 13 \%, \\
\text { monocitos: } 10 \% \text {, hemoglobina: } 7.55 \mathrm{~g} / \mathrm{dl} \text {, hematocrito: } \\
24 \% \text {, volumen corpuscular medio: } 68 \mathrm{fl} \text {, hemoglobina } \\
\text { corpuscular media: } 21 \mathrm{pg} \text {, ancho de distribución eri- } \\
\text { trocitario: } 17 \% \text {, plaquetas: } 317000 .\end{array}$ \\
Creatinina & $1.29 \mathrm{mg} / \mathrm{dL}$ \\
Nitrógeno ureico & $35 \mathrm{mg} / \mathrm{dl}\left(\mathrm{TFG} 43 \mathrm{ml} / \mathrm{min} / 1.73 \mathrm{~m}^{2}\right)$ \\
Glucemia & $133 \mathrm{mg} / \mathrm{dL}$ \\
Hierro & $6 \mathrm{umol} / \mathrm{L}$ \\
Ferritina & $22.6 \mathrm{ng} / \mathrm{ml}$ \\
Péptido natriurético tipo B & $7010 \mathrm{pg} / \mathrm{ml}$ \\
\hline
\end{tabular}

Tabla 2. Imágenes diagnósticas.

\begin{tabular}{|ll|} 
Ecocardiograma transtorácico & $\begin{array}{l}\text { Cavidades derechas con dilatación severa y altera- } \\
\text { ción moderada de la función sistólica, ventrículo } \\
\text { izquierdo dilatado, función sistólica conservada, } \\
\text { dilatación moderada de aurícula izquierda sin } \\
\text { trombos intracavitarios, esclerosis mitroaórtica } \\
\text { leve, insuficiencia mitral leve, insuficiencia tricús- } \\
\text { pidea severa por color, con velocidad que permite } \\
\text { calcular la presión arteria pulmonar sistólica en } 49 \\
\text { mmHg. }\end{array}$ \\
\hline Escanografía abdominal & $\begin{array}{l}\text { Cardiomegalia dilatada de las cuatro cavidades, } \\
\text { dilatación de vena cava, porta y supra hepáticas, } \\
\text { ascitis con distensión abdominal sin realce de } \\
\text { grasa, masa o procesos neoplásicos sugestivos, } \\
\text { ausencia quirúrgica de úteros yovarios. Hepatoes- } \\
\text { plenomegalia }\end{array}$ \\
\hline
\end{tabular}

secundaria, en el funcionamiento cardiovascular. Por último, hacen parte de este síndrome, aquellas enfermedades sistémicas que generan alteraciones a nivel cardio-renal, entre ellas las enfermedades infiltrativas, de complejos inmunes o metabólicas. Con base en los escenarios en los que se pueden presentar, el órgano primario afectado y el tiempo de evolución, se han establecido 5 tipos de SCRA (Tabla 3) (6-9).

En su magnitud, el SCRA (incluidos los SRCA) hace referencia a los efectos negativos sobre los cardiomiocitos, el endotelio, los hematíes y el complejo glomerular desencadenado ya sea por alteraciones intrínsecas, como

Tabla 3. Clasificación de los SCRA según etiología y evento secundario desencadenado (FCA: falla cardiaca aguda, ICC insuficiencia cardiaca crónica, SCA: síndrome coronario agudo, IRA: insuficiencia renal aguda, IRC: insuficiencia renal crónica, SC: shock cardiogénico DM: diabetes mellitus, LES: lupus eritematoso sistémico)

\begin{tabular}{|c|c|c|c|c|c|}
\hline & Tipo 1 & Tipo 2 & Tipo 3 & Tipo 4 & Tipo 5 \\
\hline Definición & $\begin{array}{l}\text { ICA que genera algún } \\
\text { grado de disfunción renal }\end{array}$ & $\begin{array}{l}\text { ICC que genera algún } \\
\text { grado de disfunción renal }\end{array}$ & $\begin{array}{l}\text { IRA que genera algún } \\
\text { disfunción cardiaca }\end{array}$ & $\begin{array}{l}\text { IRC que genera algún } \\
\text { grado de disfunción } \\
\text { cardiaca }\end{array}$ & $\begin{array}{l}\text { Morbilidad sistémica que } \\
\text { conlleva a disfunción } \\
\text { cardiaca y renal } \\
\text { simultáneamente }\end{array}$ \\
\hline Evento primario & ICA, SCA, SC & ICC & IRA & IRC & $\begin{array}{l}\text { DM, amiloidosis, sepsis, } \\
\text { LES, sarcoidosis }\end{array}$ \\
\hline Evento secundario & IRA & IRC & $\begin{array}{l}\text { ICA, SCA, arritmia, } \\
\text { shock }\end{array}$ & IRC, FCA, SCA & $\begin{array}{l}\text { ICA, ICC, SCA, IRA, } \\
\text { IRC, SC }\end{array}$ \\
\hline Prevalencia & $30 \%$ & $57 \%$ & $9 \%,(35 \% \mathrm{UCI})$ & $11 \%$ & desconocida \\
\hline
\end{tabular}


extrínsecas cuyas complicaciones generan disfunción de estos órganos. La lesión que desencadena la activación del sistema renina-angiotensina-aldosterona, péptido natriurético, y vasopresina, se ve exponencialmente exacerbada por el arsenal proinflamatorio de citoquinas, que condenan los insuficientes mecanismos compensadores a fracasar.

$\mathrm{La}$ anemia en el curso de la insuficiencia cardiaca (ICC) se ha documentado como comorbilidad en pacientes con edad avanzada, diabetes mellitus (DM), insuficiencia renal crónica (IRC), disnea con clase funcional avanzada (NYHA 3/4), capacidad reducida para hacer ejercicio, edema, mala calidad de vida, entre otras. Anteriormente se relacionaba la hemoglobina $(\mathrm{Hb})$ y la fracción de eyección del ventrículo izquierdo (FEVI) como agentes que guardaban una relación directamente proporcional, pero en la actualidad se sabe que a menor concentración de $\mathrm{Hb}$ en sangre, hay mayor compromiso de la FEVI, pero su incremento, paradójicamente, genera un impacto negativo en la función cardiovascular. La etiología de la anemia es multifactorial: la malabsorción por la hipoperfusión esplácnica, las pérdidas gastrointestinales por el uso de antiagregantes plaquetarios o anticoagulantes, y el estado inflamatorio sistémico constituyen sus principales contribuyentes (Figura 1). Con la finalidad de aumentar el trasporte y la entrega de oxígeno, en el sistema hematopoyético se llevan a cabo una serie de mecanismos compensadores, siendo el aumento en la eritropoyesis, de los glóbulos rojos y del 2,3 difosfoglicerato los más representativos.

A nivel endotelial y vascular, hay una disminución de la resistencia vascular sistémica (RVS) y de la viscosidad, con hipotensión secundaria empeorada por la aumentada expresión de óxido nítrico (ON). Todos estos cambios neurohumorales impactan morfológicamente las estruc- turas ventriculares y glomerulares que soportan el nocivo circulo vicioso (10-13).

El tratamiento debe ser multidisciplinario, basado en la fisiopatología bidireccional cardio-renal y renocardiaca. Cuando el órgano primario que se afecta es el corazón, se debe instaurar tratamiento tanto para evento agudo en el SCRA tipo 1 (con diurético, inotropía, asistencia ventricular, etc.), como para el crónico en el SCRA tipo 3 (con inhibidor de la ECA, antagonistas de receptores angiotensina, antagonistas de aldosterona, estatinas, entre otras). Cuando la noxa primaria se documenta en el riñón, se debe instalar el tratamiento: en el SCRA tipo 2 y 4 , el manejo es similar al descrito previamente, pero se adiciona la opción de ultrafiltración o remoción de la volemia mediante terapia dialítica, mientras que el manejo para el SCRA tipo 5 se fundamenta en control de la enfermedad de base, adjunto a terapia sobre factores de riesgo. La anemia, que desempeña un papel fundamental en el SCRA, debe manejarse agresivamente con hierro IV y eritropoyetina recombinante, dado que se ha demostrado la mejoría de la función renal, medida en tasa de filtración glomerular y la mejoría en la función cardiaca, con disminución o detención de cambios de remodelación a nivel ventricular (14-18).

El manejo de los pacientes que cursan con esta patología debe ser integral, dirigido a la intervención de las alteraciones de base, para así controlar la sobreexpresión de agentes neurohumorales y citoquinas proinflamatorias, dirigido por un grupo de trabajo que conozca las interacciones cardiorenales, las complicaciones y las asociaciones, para asi instaurar tratamientos dirigidos al problema en su magnitud, siendo un reto para el internista actual, desde su diagnóstico hasta su manejo, el inadvertido síndrome cardio-renal anémico.

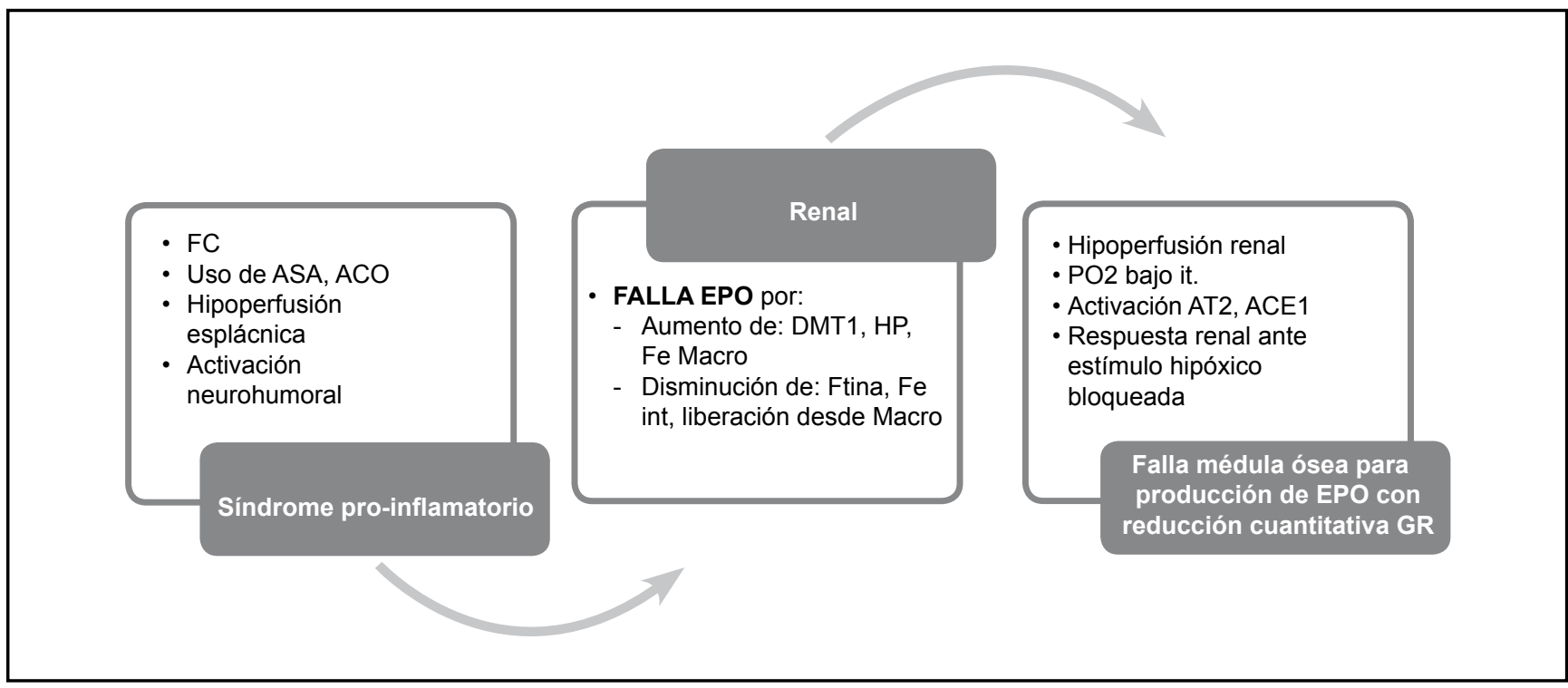

Figura 1. Fisiopatología anemia en Falla Cardiaca crónica (FC: falla cardiaca, EPO: eritropoyesis, DMT1: trasportador divalente, HP: hepcidina, Fe: hierro, MACRO: macrófagos, FTINA ferritina, Fe int: hierro intestinal, PO2: presión de oxígeno, AT2: angiotensina 2,ACE1: enzima convertidora de angiotensina 1,GR: glóbulos rojos).

Adaptado de "Anemia And Chronic Heart Failure Implications And Treatment Options, Anand". 


\section{Referencias}

1. Mentz, RJ, Lewis EF. Epidemiology of cardiorenal syndrome. Heart Failure Clinics 2010; 6: 333-46.

2. Ahmed et Campbell. Epidemiology of chronic kidney disease in heart failure. Heart Failure Clinics 2008; 4: 387-99.

3. Tang WH Wilson, Yeo PS Daniel. Epidemiology of anemia in heart failure. Heart Failure Clinics 2010; 6: 271-8,

4. Amsalem, Garty, Schwartz et al. Prevalence and significance of unrecognized renal insufficiency in patients with heart failure. European Heart Journal 2008; 29: 1029-36.

5. Coresh, Astor, Greene, Eknoyan et Levey. Prevalence of chronic kidney disease and decreased kidney function in the adult us population: third national health and nutrition examination survey. American Journal Kidney Disease 2003; 41: $1-12$.

6. Ronco, Haapio House et al. Cardiorenal syndrome. J Am Coll Cardiol 2008; 52: $1527-1539$.

7. Ronco, Maccullough, Anker, et al. Cardio-renal syndromes: report from the consensus conference of the acute dialysis quality initiative. Eur Heart J 2010; 6: 703-11.

8. Mahapatra, Lalmalsawma, Singh, Kumar, Tiwari. Cardiorenal syndrome. Iran Journal Kidney Disease 2009; 3: 61-70.

9. Pokhrel Narayan, Maharjan Najindra, Dhakal Bismita, Arora R Rohit. Cardiorenal syndrome: A literature review. Exp Clin Cardiol 2008; 13(4): 165-70.
10. Kazory et Ross. Anemia: the point of convergence or divergence for kidney disease and heart failure. Journal of The American College of Cardiology 2009: $53(8)$.

11. Sandhu, Soman, Hudson et Besarab. Managing anemia in patients with chronic heart failure: what wdo we know? Vascular Health And Risk Management 2010: 6: $237-52$

12. Taring. Cardiorenal anemia syndrome in chronic kidney disease. Journal Chinn Med Assoc 2007; 70(10): 424-429

13. Anand, Anemia and chronic heart failure implications and treatment options. Journal of The American College of Cardiology 2008; 52(7).

14. Kazory. Need for a unified decision-making tool for ultrafiltration therapy in heart failure; call for action. American Heart Journal 2010;159:505-7

15. Jie, Verhaar, Maarten-Jan, et al. Erythropoietin and the cardiorenal syndrome: cellular mechanisms on the cardiorenal connectors. 291:F932-F944, $2006 \mathrm{Am} \mathrm{J}$ Physiol Renal Physiol

16. Paluzzuoli, Silverberg, Iovine, et al. Effects of b-erythropoietin treatment on left ventricular remodeling, systolic function, and B type natriuretic peptide levels in patients with the cardiorenal anemia syndrome. American Heart Journal 2007; 154: 645, E9-645e15.

17. Besarab, Hörl et Silverberg. Iron metabolism, iron deficiency, thrombocytosis, and the cardiorenal anemia syndrome. The Oncologist 2009; 14(Suppl 1): 22-33.

18. House, Haapio, Lassus, Bellomo et Ronco. Therapeutic strategies for heart failure in cardiorenal syndrome. American Journal Of Kidney Diseases 2010; 4: 759-773. 